\title{
Polyisobutylene-block-poly(methacrylic acid) Diblock Copolymers: Self-Assembly in Aqueous Media
}

September 26, 2007 
Markus Burkhardt ${ }^{1}$, Nemesio Martinez-Castro ${ }^{1}$, Sandrine Tea ${ }^{1}$, Markus Drechsler ${ }^{1}$, Ivan Babin ${ }^{2}$, Ivan Grishagin ${ }^{2}$, Ralf Schweins ${ }^{3}$, Dmitry V. Pergushov ${ }^{2}$, Michael Gradzielski ${ }^{4}$, Alexander B. Zezin ${ }^{2}$, Axel H.E. Müller ${ }^{*}$

${ }^{1}$ Makromolekulare Chemie II, Universität Bayreuth, D-95440 Bayreuth, Germany;

${ }^{2}$ Department of Polymer Science, School of Chemistry, Moscow State University, 119991 Moscow, Russia;

3 Institut Laue-Langevin, 38042 Grenoble Cedex 9, France;

${ }^{4}$ Stranski Laboratorium für Physikalische und Theoretische Chemie, Institut für Chemie, Technische Universität Berlin, D-10623 Berlin, Germany;

* e-mail: axel.mueller@uni-bayreuth.de

Markus Burkhardt: markus.burkhardt@uni-bayreuth.de;

Nemesio Martinez-Castro: nemesiomc@yahoo.com;

Sandrine Tea: sandrine.tea@uni-bayreuth.de;

Markus Drechsler: markus.drechsler@uni-bayreuth.de;

Ivan Babin: babin_ia@mail.ru;

Ivan Grshagin: engewo@yahoo.com;

Ralf Schweins: schweins@ill.fr;

Dmitry V. Pergushov: pergush@genebee.msu.su;

Michael Gradzielski: michael.gradzielski@tu-berlin.de;

Alexander B. Zezin: zezin@genebee.msu.su; 


\section{Supporting Information}

\section{$1.1 \quad$ Synthesis}

The $\mathrm{PIB}_{x}-b-\mathrm{PMAA}_{y}$ used for the investigations in this work are shown in Table 1. The hydrophobic PIB block used as a macroinitiator as described elsewhere [1] was obtained by quenching the cationic polymerization of IB with thiophene. The PDI of approximately 1.12 to 1.19 are relatively low for cationic polymerization. For this work three PIB macroinitiators with $\overline{D P_{n}}$ of 25,30 and 75 where utilized as precursors for the amphiphilic diblock copolymers. Anionic polymerization resulted in rather monodisperse polymers of PDI of 1.03 to 1.14, except for polymer "3" with a PDI of 1.22 , which is still narrow.

In Figure 1 representative GPC traces of $\mathrm{PIB}_{75}-b-\mathrm{P} t \mathrm{BMA}_{1600}$ are shown, with the dashed lines, representing the UV signal and the dotted lines, representing the RI response of the apparatus. For the $\mathrm{PIB}_{75}$ precursor, the UV trace shows a small shoulder at higher $M_{n}$. The shoulder is placed at the position corresponding to double molecular weight of the main peak, suggesting coupling of two cationic PIB chains to one thiophene, as already reported by Martinez-Castro et al. [1]. The fact that this shoulder is not observed in RI trace implies that the amount of this double molecular weight product is not too high. The pronounced response in UV-trace is due to the strong response of the aromatic thiophene incorporated in the chain. A second PIB chain blocks the 5-position of the thiophene ring, hence 


\begin{tabular}{|c|c|c|c|c|}
\hline Polymer & $\overline{D P_{n}}, \mathrm{PIB}$ & $\overline{D P_{n}}, \mathrm{PtBMA}$ & $\begin{array}{c}\overline{M_{n}}(\mathrm{GPC}) \\
\frac{g}{m o l}\end{array}$ & $\begin{array}{c}P D I \\
(\mathrm{GPC})\end{array}$ \\
\hline PIB 1 & 25 & - & 1,340 & 1.16 \\
\hline PIB 2 & 30 & - & 1,740 & 1.19 \\
\hline PIB 3 & 75 & - & 4,270 & 1.12 \\
\hline 1 & 75 & 615 & 57,000 & 1.05 \\
\hline 2 & 75 & 1600 & 141,000 & 1.03 \\
\hline 3 & 25 & 350 & 31,600 & 1.22 \\
\hline 4 & 75 & 190 & 20,500 & 1.09 \\
\hline 5 & 25 & 2600 & 222,000 & 1.02 \\
\hline 6 & 30 & 190 & 18,000 & 1.14 \\
\hline 7 & 30 & 170 & 16,400 & 1.12 \\
\hline
\end{tabular}

Table 1: Molecular characteristics of the $\mathrm{PIB}_{x}-b$-PtBMA $y$ used in this work it cannot be activated by BuLi for initiating the polymerization of $t$ BMA.

The GPC traces obtained after purification of the diblock copolymer (Figure 1) show monomodal distribution. At 32 - $34 \mathrm{ml}$ elution volume, almost no precursor is left, except of a tiny peak that can be attributed to coupled PIB that could not initiate anionic polymerization of $t$ BMA. After elimination of $\mathrm{t}$-Bu moieties via hydrolysis of PtBMA blocks with hydrochloric acid in dioxane at $80^{\circ} \mathrm{C}$, PIB- 


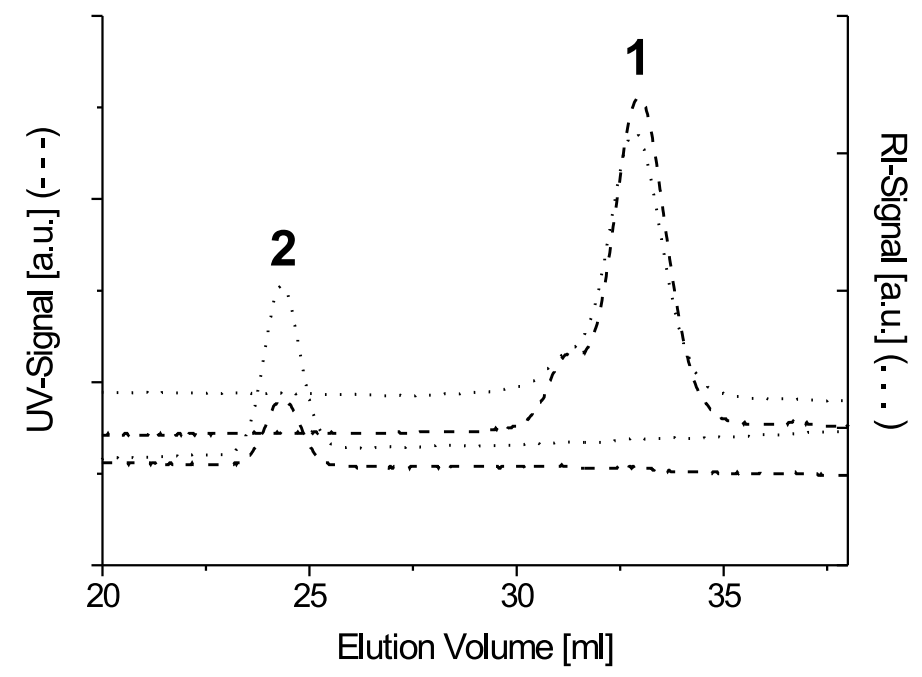

Figure 1: GPC traces of the $\mathrm{PIB}_{75}$ precursor (1) for anionic polymerisation and purified $\mathrm{PIB}_{75}-b$-PtBMA 1600 block copolymer (2) obtained in THF; $(\cdots)$ : RI-signal; (- - ): UV-signal $(\lambda=260 \mathrm{~nm})$. 
b-PMAA diblock copolymer was obtained. The degree of hydrolysis obtained by means of IR spectroscopy provides evidence for nearly complete conversion (i, 95 \%). The coupled PIB can be removed from PIB-b-PMAA by precipitation of this diblock copolymer into hexane [2].

\section{References}

[1] Martinez-Castro, N.; Lanzendörfer, M.G.; Müller, A.H.E.; Cho, J.C.; Acar, M.H.; Faust, R.; Macromolecules, 2003, 36, 6985;

[2] Feldthusen, J.; Thesis, 1998, Makromolekulare Chemie II, Bayreuth; 